phase, after a 4-8 week lead-in period. Daily dose beginning at $25 \mathrm{mg}$ was increased in steps of $25 \mathrm{mg}$ every week to $100 \mathrm{mg}$. A further increase to $200 \mathrm{mg} / \mathrm{day}$ was made if required, but the dose remained stable for the last 4 weeks of treatment. Patients were randomly assigned to continue topiramate or switch to placebo for a 26-week double-blind phase. The number of days with migraine during the last 4 weeks of the double-blind phase was compared with the last 4 weeks of the open-label phase. The mean increase in number of migraine days was greater in the placebo group than in the topiramate group. Acute medication was used more frequently by the placebo than the topiramate group. Quality of life remained stable and patients were more satisfied with treatment efficacy while on topiramate compared to placebo. Tolerability was similar in both groups. Sustained benefit was obtained after discontinuing topiramate 6-month trial, but number of migraine days showed an increase. Patients should be treated for 6 months as a general rule, with the option to continue to 12 months in some patients. (Diener H-C, Agosti R, Allais G, et al. Cessation versus continuation of 6-month migraine preventive therapy with topiramate (PROMPT): a randomized, double-blind, placebo-controlled trial. Lancet Neurol December 2007;6:10541062). (Respond: Hans-Christoph Diener MD, Department of Neurology, University Duisburg-Essen, 45122 Essen, Germany).

COMMENT. This study of migraine prophylactic treatment included greater numbers of patients and was of longer duration than most previous trials. The authors hypothesize that the long-term effect of topiramate might be to correct the neuronal dysfunction factor in migraine pathophysiology. Since frequent migraine might lower the threshold for future attacks and promote development of chronic migraine, long-term prophylactic therapy should be considered as an option in adolescents with recurrent severe attacks.

The pros and cons of preventive compared to abortive antimigraine treatment are discussed in an editorial (Couch JR Jr. Preventive antimigraine therapy: the long-term outlook. Lancet Neurol Dec 2007;6:1035-1036). What are the indications for preventive therapy? Is the improvement worth the potential adverse effects? Does tachyphylaxis develop with long-term treatment? How quickly should treatment be withdrawn? Does preventive therapy lessen or increase the incidence of chronic daily headache? The results of the above study indicate that rapid withdrawal of topiramate caused a rebound headache occurrence, and future trials should lengthen the period of withdrawal. The data support the conclusion that preventive topiramate therapy has a residual beneficial effect, resetting the migraine cycle and raising the threshold.

\title{
IV DEXAMETHASONE FOR ACUTE MIGRAINE
}

A randomized trial of dexamethasone $10 \mathrm{mg}$ IV in 205 adult patients presenting with acute migraine to an emergency department (ED) found $25 \%$ of those with acute migraine became pain-free for at least 24 hours compared to $19 \%$ of those given placebo $(p=0.34)$. Patients with status migrainosus (migraine lasting longer than 72 hours) received persistent relief in $38 \%$ compared to $13 \%$ following placebo $(\mathrm{p}=0.06)$. All patients also received IV metoclopramide and diphenhydramine as pimary treatment for migraine. Researchers from the Department of Emergency Medicine (ED), Albert Einstein College of Medicine, Montefiore Medical Center, Bronx, NY, advise that IV dexamethasone should not be administered routinely in ED treatment of acute migraine, but it might be considered for 
patients with migraine lasting longer than 72 hours. (Friedman BW, Greenwald P, Bania TC, et al. Randomized trial of IV dexamethasone for acute migraine in the emergency department. Neurology Nov 27, 2007;69(22):2038-2044). (Reprints: E-mail: befriedm@montefiore.org ).

COMMENT. The authors comment that the benefit in persistent migraine relief rates found for dexamethasone in this study should be interpreted with caution because this was a subgroup analysis. Becker WJ, Kryscio RJ, in an editorial (Neurology 2007;69:2034-2035), note that others have reported benefits of dexamethasone, especially with larger doses and with headache endpoints beyond 24 hours post-treatment.

\section{SEIZURE DISORDERS}

\section{LONG-TERM SOCIAL OUTCOMES IN CHILDHOOD EPILEPSY}

Population-based longitudinal and cross-sectional studies of social outcomes of children with epilepsy in different countries are reviewed by researchers at Dalhousie University, Halifax, Nova Scotia, Canada. The Isle of Wight UK study (Rutter et al, 1970) found psychiatric problems in $25 \%$ of children with epilepsy compared to $7-9 \%$ of normal controls. A Finnish series of children with epilepsy followed to 22 years of age had severe learning disorders in $20 \%$ compared to $2 \%$ of controls (Kokkonen et al, 1997). At longer follow-up, patients were more likely on disability pension ( $12 \%$ vs $2 \%$ controls), twice as likely to be unemployed, and more often unmarried, living alone or with parents (69\% vs $40 \%$ controls). Cognitive or neurologic disability accounted for the poor social outcome, and seizure control and socioeconomic status were unrelated to the outcome. In Japan (Wakamoto et al, 2000), unlike Finland, normally intelligent young adults with epilepsy had favorable long-term educational and employment outcomes, but the mentally retarded did poorly. In Turku, Finland (Sillanpaa, 1990; Jalava et al, 1997), adults with childhood epilepsy had reduced levels of education, employability, social economic status, and successful marriage $(p=0.0001)$, and $15 \%$ were dependent on other caretakers. Those on AEDs were less satisfied with their quality of life than those off medication.

In the Canadian Nova Scotia, population-based epilepsy cohort study (Camfield et al, 1993, 2007; Wirrell et al, 1997), poor social outcome was more common in patients with a learning disorder. Young adults with childhood absence epilepsy had less education, lower working status and higher rates of behavioral and psychiatric difficulty than a chronic disease control group with juvenile rheumatoid arthritis. Children with secondary generalized epilepsy were often mentally handicapped and/or highly dependent with refractory seizures. (Camfield CS, Camfield PR. Longh-term social outcomes for children with epilepsy. Epilepsia December 2007;48(Suppl.9):3-5). (Respond: Carol S Camfield MD, FRCP(C), IWK Health Centre, PO Box 9700, Halifax, Nova Scotia, Canada B3K 6R8. E-mail: Camfield@dal.ca).

COMMENT. Learning disorder and mental handicap are the most significant risk factors for poor social outcome in adults with a history of childhood epilepsy. The effect of epilepsy on social outcome is greater than that of other childhood chronic disease. 